\title{
VLBA PHASE-REFERENCING
}

\author{
A.J. BEASLEY, J.E. CONWAY and P.J. DIAMOND \\ National Radio Astronomy Observatory, \\ P.O. Box 0 Socorro NM USA 87801. * \\ W.D. COTTON \\ National Radio Astronomy Observatory, \\ Edgemont Rd., Charlottesville VA USA 22903. \\ and \\ R.C. VERMEULEN \\ California Institute of Technology, Pasadena CA USA 91125. **
}

\begin{abstract}
Residual delay errors in VLBI data limit the image sensitivity and dynamic range at tainable using current calibration techniques such as self-calibration. Applying phase corrections derived from regular observations of nearby calibrators (phase-referencing), the residual delay errors on a target source can be reduced, increasing coherent integration times. In this paper we present the results of a series of observations made with the VLBA to examine the effectiveness of phase-referencing for improving instrumental phase stability. Optimum observing strategies when studying weak $(\sim \mathrm{mJy})$ sources are discussed.
\end{abstract}

Key words: Phase-referencing - VLBA - calibration - atmosphere

The phase measured by a radio interferometer contains the effects on wavefront propogation of the ionosphere, the wet and dry troposphere, and the receiving electronics; systematic errors due to inaccurate geometry (e.g. source/station position errors \& antenna axis offsets) and clock errors are also included. Using selfcalibration (e.g. fringe-fitting) these errors can be reduced to some extent, however: (1) absolute position information is lost; and (2) typical self-cal procedures recuire sufficient signal-to-noise to identify a solution in delay/delay-rate space (typically $\mathrm{S} / \mathrm{N} \sim 7)$.

One strategy to remove these constraints for VLBI observations, termed phasereferencing, involves regular switching to a strong nearby (typically $\leq 5^{\circ}$ ) reference calibrator to determine a phase correction to be applied to the target source. This process removes the effects of any clock errors, and reduces the effects of geometry and atmospheric variations by a factor roughly equal to $1 /($ target-reference separation in radians). The result is a increase in the coherent integration time used for determining self-cal corrections, or sufficient phase stability to enable direct imaging of the data (i.e. fourier inversion and CLEANing). There are, however, a number of parameters related to phase-referencing that are not yet clearly known, e.g. the optimal target-reference separation and switching-cycle time, the effects of temporal and spatial variations in the atmosphere, and the overall success rate of phase-referencing in improving phase stability at different frequencies. In this paper we present some results from a series of VLBA 8.4 and $1.6 \mathrm{GHz}$ observations using pairs of calibrator sources of various separations to examine some of these parameters.

* The NRAO is operated by Associated Universities, Inc., under agreement with the National Science Foundation.

** This work was supported in part by the NSF under grant AST-9117100 
The observations to date are summarized in Table 1; Sep is the source separation in degrees, $\mathrm{N}_{\text {obs }}$ the number of observations. Each observation consists of $1 \mathrm{hr}$ at both $8.4 \mathrm{GHz}$ and $1.6 \mathrm{GHz}$ with a 6 minute switching-cycle time (i.e. 3 mins on src A, 3 minutes on src B); typically 3-6 antennas were used. The observations were made between $1992 \mathrm{~J}$ an 17 and $1992 \mathrm{Jul} 14$ using the MKII recording system, the tapes correlated on the NRAO MKII processor. We have written an AIPS task to recalculate delays and apply phase-corrections to VLBI data, at present based on the UVGEOM code (Conway \& Vermeulen 1993, in preparation).

\section{TABLE I}

VLBA Phase-reference data

\begin{tabular}{ccc}
\hline Src pairs (T/R) & $\operatorname{Sep}\left({ }^{\circ}\right)$ & $\mathrm{N}_{\text {obs }}$ \\
\hline $1222+037 / 1226+023$ & 1.9 & 6 \\
$1611+343 / 1641+399$ & 8.9 & 4 \\
$0913+391 / 0923+392$ & 0.7 & 2 \\
$1638+398 / 1641+399$ & 0.3 & 1 \\
\hline
\end{tabular}

After correlation, the data were read into AIPS where the antenna-earth centre delay for each data point are recalculated; at present, we incorporate corrections for source/station positions, polar wobble, antenna axis offsets, and the wet+dry troposphere delay. These delays are then compared to those used at correlation time (as written in the CL table), and an appropriate phase correction inserted. The reference source is then fringe-fitted, and the resultant phase corrections are interpolated to the target source.

Some typical phase-referencing results are shown in Fig. 1, from the observation $1992 \mathrm{~J}$ an $17 / 1222+037-1226+023 / 8.4 \mathrm{GHz}$. The typical residual fringe rate before phase-referencing was $\sim 5 \mathrm{mHz}$; Fig. 1 shows the target residual phases after phasereferencing on the calibrator source. The phases are stable over $1 \mathrm{hr}$, with residual fringe rates $\ll 0.3 \mathrm{mHz}$.

\section{Results}

Summarizing our findings for both frequencies:

- $\quad$ 8.4 GHz - Coherence times $\gg 1$ hr were obtained $100 \%$ of the time $(6 / 6$ obs) at $1.9^{\circ}$ separation on baselines ranging from $\sim 200-5000 \mathrm{~km}$. Equivalent phase stability was found only $50 \%$ of the time $(2 / 4)$ at $8.9^{\circ}$ separation.

- $\quad 1.6 \mathrm{GHz}$ - Coherence times of $\sim 20-30$ mins were obtained $66 \%$ of the time $(4 / 6)$ at $1.9^{\circ}$ separation, and $50 \%$ of the time at $8.9^{\circ}$. A coherence time $\gg 1 \mathrm{hr}$ was obtained for the $0.3^{\circ}$ separation observation. In most cases, the phase stability was limited by the large $\left(\sim 100^{\circ}\right)$ coherent variations possibly due to travelling ionospheric disturbances (TIDs, discussed below).

In Fig. 2(a,b) examples of the $1.6 \mathrm{GHz}$ phase stability are shown; these data are for $0.3^{\circ}$ and $8.9^{\circ}$ separations, observed at a fairly low elevation for $1611+343\left(\sim 20^{\circ}\right)$. 


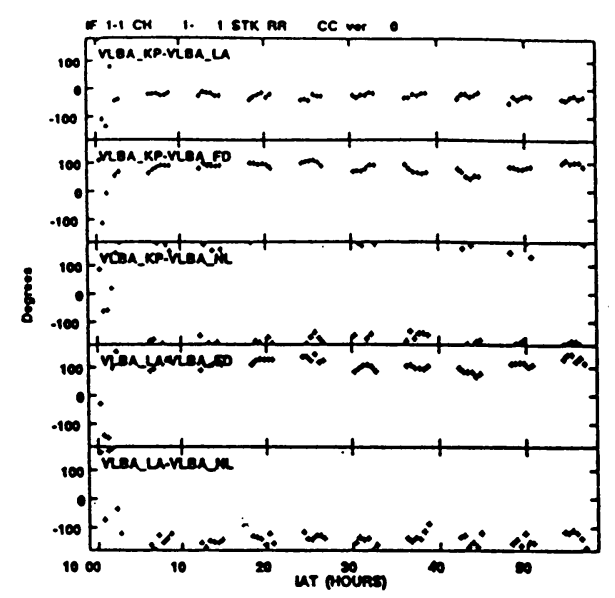

Fig. 1. Target residual phases after phase-referencing. Coherence time $\gg 1 \mathrm{hr}$.

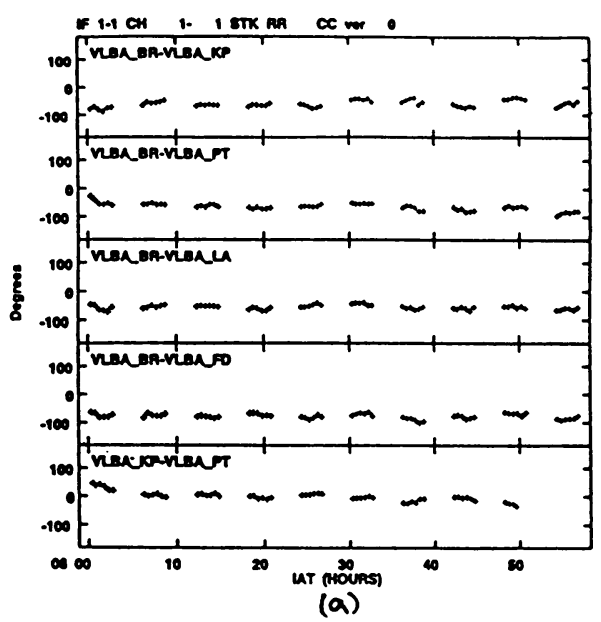

Fig. 2. Target residual phases using $0.3^{\circ}(\mathrm{a})$ and $8.9^{\circ}$ calibrator.

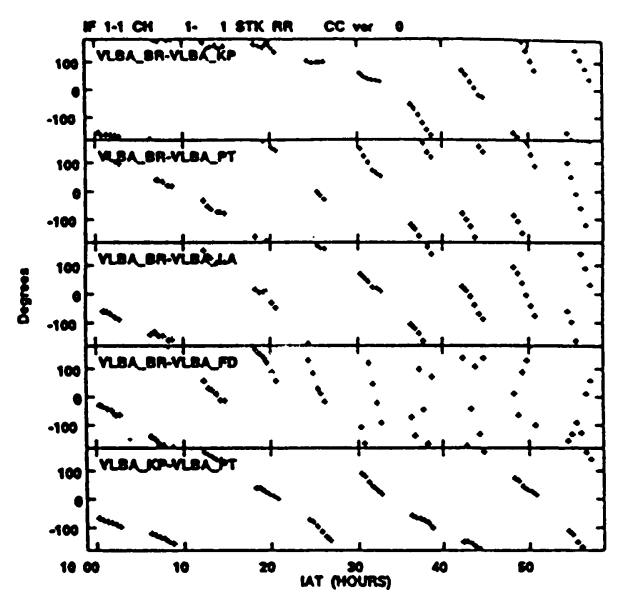

(b)

(b) separation phase-reference

During the atmospheric/ionospheric conditions prevalent during this observation a reference source $8.9^{\circ}$ away appears unsatisfactory at both 8.4 and $1.6 \mathrm{GHz}$ for significantly improving coherence times. 
The major source of residual error after phase-referencing the $8.4 \mathrm{GHz}$ data appears to be errors in the estimation of the zenith delays due to the ionosphere and troposphere; for example, the tropospheric delay (assumed to be $\sim 2.1 \mathrm{~m}$ here) can be estimated only to a $2 \mathrm{~cm}$ accuracy (Elgered 1982, IEEE Tran. Ant. \& Prop., AP30, 502). Such errors lead to slowly varying ( few hrs) phase residuals such as those seen in Conway \& Vermeulen (1993). Errors in zenith delays lead to differential phase residuals due to the different elevations of the target and reference sources, i.e. the assumption of isoplanicity breaks down.

Our results seem to indicate that a target-reference separation of $\sim 2^{\circ}$ is sufficient for phase-referencing to increase coherence times to $\gg 1 \mathrm{hr}$ at $8.4 \mathrm{GHz}$. The distribution of flat-spectrum phase-calibration sources with flux-densities above $200 \mathrm{mJy}$ described by Patnaik et al. (1992, MNRAS, 254,655) indicates a $71 \%$ chance of a reference source within $2^{\circ}$ of any target source, and $94 \%$ within $3^{\circ}$. Our simulations suggest that after phase-referencing, target sources above $\sim 2 \mathrm{mJy}$ can be self-calibrated, while sources below $2 \mathrm{mJy}$ can be directly imaged as long as the zenith path delay can be estimated within $\sim 1 \lambda$. For both 8.4 and $1.6 \mathrm{GHz}$, a switching-cycle time of 6 mins appears sufficient for elevations above $20^{\circ}$ to allow phase connection (i.e. there is no $2 \pi$ ambiguity in phase interpolation), although there are exceptions (e.g. Conway \& Vermeulen 1993).

The success of phase-referencing at $1.6 \mathrm{GHz}$ is more limited due to the variable components of the ionosphere such as TIDs. Variations in electron density and therefore ionospheric delay lead to spatial gradients in delay, a typical example of which may be séen in Fig. 2(b) (see van Velthoven 1990, Ph.D. Thesis, Univ. Eindhoven). Smaller target-reference separations (e.g. $\leq 1^{\circ}$ ?) may be need to significantly improve coherence times beyond 10-20 mins. The static ionospheric zenith delay can be more accurately derived from multi-wavelength data (i.e. "S/X" calibration).

\section{Future Work}

We are currently testing an implementation of the Goddard CALC program to recalculate delays, which should provide $\sim 1$ picosecond delay accuracy using more accurate geometrical and atmospheric modelling (currently the VLBA correlator is using CALC for delay modelling). Further modelling of the effects of the ionosphere and TIDs on VLBI data is also underway. We are also pursuing more extensive observations to explore the effects of different separations, elevations and weather on the phase-referencing technique, including longer tracks on source pairs to investigate longer-term (i.e. $12 \mathrm{hr}$ ) residual errors.

\section{Discussion:}

\section{Napier:}

For VLBI phase referencing, how big a problem is resolved structure in most of the calibrators, and what is the solution?

Beasley:

Significant structure in the calibrator source will need to be modelled as part of the phase-referencing process. A cycle of self-calibration and mapping will be required when using reference sources with inaccurate positions and/or structure. 